\title{
Centre-to-limb variation of solar granulation in the infrared
}

\author{
M. Sánchez Cuberes ${ }^{1}$, M. Vázquez ${ }^{1}$, J. A. Bonet ${ }^{1}$, and M. Sobotka ${ }^{2}$ \\ 1 Instituto de Astrofísica de Canarias, 38205 La Laguna, Tenerife, Spain \\ e-mail: msanchez@ll.iac.es; mva@ll.iac.es; jab@ll.iac.es \\ 2 Astronomical Institute, Academy of Sciences of the Czech Republic, 25165 Ondřejov, The Czech Republic \\ e-mail: msobotka@asu.cas.cz
}

Received 25 January 2002 / Accepted 22 October 2002

\begin{abstract}
Broad-band images have been obtained at the extremes of the continuum absorption coefficient of the solar atmosphere, at $\lambda 0.8 \mu \mathrm{m}$ and $\lambda 1.55 \mu \mathrm{m}$. Quiet regions have been observed for different heliocentric angles. The centre-to-limb variation of granulation contrast has been computed in both wavelength ranges and a steeper decrease in the contrast is obtained for $\lambda 0.8 \mu \mathrm{m}$ than for $\lambda 1.55 \mu \mathrm{m}$. For positions near the solar limb the granulation contrast shows a tendency to increase at both wavelengths. Mean granular sizes vary from $1.25 \operatorname{arcsec}^{2}$ at the disc centre to $2 \operatorname{arcsec}^{2}$ at $\mu=0.6$ in the $\lambda 0.8 \mu \mathrm{m}$ images and from $1.24 \operatorname{arcsec}^{2}$ to $1.85 \operatorname{arcsec}^{2}$ at $\lambda 1.55 \mu \mathrm{m}$. Observations close to the limb detect granular structures of 0 ' $^{\prime} 96$ or even smaller at a distance from the limb of $d=0$ ' 32 , equal to the diffraction limit of the telescope at $\lambda 8000 \AA$. Using an Eddington-Barbier approximation this implies a penetration of the temperature fluctuations associated with granulation up to approximately $z \approx$ $220 \mathrm{~km}$, although the calculation of response functions defines a broader interval.
\end{abstract}

Key words. Sun: granulation - Sun: photosphere

\section{Introduction}

For simple geometrical reasons the information that we receive when observing at different heliocentric angles comes from different heights in the solar atmosphere. Many photometric investigations have profited from this fact to study the vertical structure of the solar photosphere (e.g. Schmidt et al. 1979; Durrant et al. 1983; Wilken et al. 1997). Nevertheless, the results vary widely from one author to another, with no possibility of finding a clear correlation between the different methods used, observing wavelengths and image treatment techniques (Sánchez Cuberes et al. 2000). The study of the centre-to-limb variation (CLV) of granulation contrast allows us to infer information about the change with height in the horizontal temperature differences associated with the granulation. The characterization of this dependence is of crucial importance for understanding the mechanisms that underly the penetration of overshooting granules into the photosphere and set boundary conditions on the models of solar convection.

In this paper we perform a study of the CLV of granulation in the infrared (IR), near the two extremes of the continuum absorption coefficient of the solar atmosphere. This technique has been recently applied by the authors to study facular regions (Sobotka et al. 2000; Sánchez Cuberes et al. 2002). The study at $1.55 \mu \mathrm{m}$ is especially interesting since it allows us to observe the photosphere at the deepest observable layers,

Send offprint requests to: M. Sanchez Cuberes, e-mail: msanchez@ll.iac.es where the 5-min oscillations contribution to the measured granulation contrast (see Koutchmy \& Lebecq 1986) is considerably reduced. In parallel, the effects of atmospheric seeing (see Koutchmy 1994; Keil et al. 1994), and line blocking are reduced, thereby allowing a higher precision in the measurements. Rao et al. (2001) study how the granular contrast is affected by several instrumental and atmospheric parameters.

The difference in formation height of $1.55 \mu \mathrm{m}$ and $0.8 \mu \mathrm{m}$ observations is approximately 30 or $40 \mathrm{~km}$ (see Sect. 3). However, the temperature fluctuations decrease very rapidly between these two layers, so that the IR observations seem to be more useful in setting upper boundary conditions to the models of small-scale solar convection.

On the other hand, IR observations suffer the disadvantage that granulation shows a smaller contrast and telescopes have less spatial resolution at these wavelengths, constraints that we will keep in mind throughout this paper.

2-D power spectra of granulation images at the opacity minimum show more power at high spatial frequencies accompanied by a reduction at the lower ones as compared to observations performed at shorter wavelengths (Koutchmy 1990). CLV curves of the rms contrast have been published by Turon \& Lena (1973) and Koutchmy (1994), but limited to the spatial range $1.0>\mu>0.7$. At IR wavelengths larger than $1.6 \mu$ the radiation is coming from progresively higher layers. Observations at $12.4 \mu$ show more rapid temporal changes than in the visible and the lack of the cell-like character (Gezari et al. 1999). 


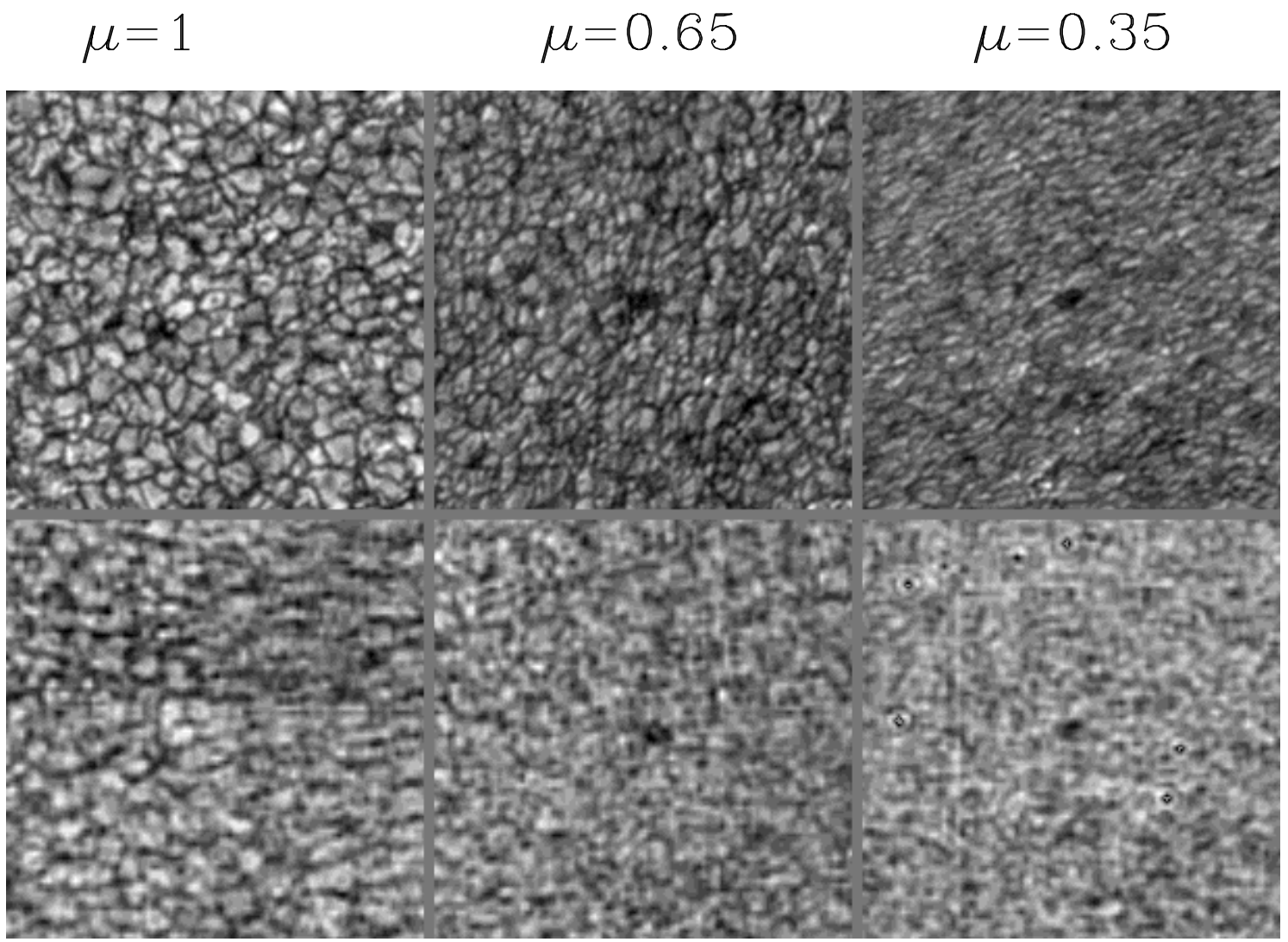

Fig. 1. Pairs of nearly simultaneous images of the granulation at $\lambda 0.8 \mu \mathrm{m}$ (upper panels) and $\lambda 1.55 \mu \mathrm{m}$ (lower panels) at three $\mu$-positions. The boxes cover a field of $33^{\prime \prime} .32 \times 33^{\prime \prime} 32$.

Taking advantage of the good resolution of the images acquired, a photometric study of the CLV of granular sizes is also performed here. These kind of studies are useful for understanding the physical scales on which the granulation takes place.

The height penetration of overshooting granules has been extensively studied by means of spectroscopic data, and different authors have obtained different values for the height where the granulation vanishes, with the associated velocities penetrating higher in the photosphere than the corresponding temperature fluctuations. Here, we will perform a photometrical study of granulation at the extreme limb, which can produce new information on this controversial topic.

\section{Observations}

During the observing campaign 1997 June 17-29 at the Swedish Vacuum Solar Telescope (SVST, Observatorio del Roque de los Muchachos, La Palma - see Scharmer et al. 1985) broad-band images of quiet regions were obtained at various heliocentric angles. They were taken simultaneously in two channels:

1) $\lambda=0.8000 \pm 0.0025 \mu \mathrm{m}$ (continuum opacity maximum) with a 10-bit $1536 \times 1032$ pixel CCD Kodak Megaplus 1.6. The image scale and exposure time were $00^{\prime} 083$ pixel $^{-1}$ and $25 \mathrm{~ms}$, respectively. The camera was working in real-time frame selection mode with selection intervals of $20 \mathrm{~s}$.
2) $\lambda=1.5542 \pm 0.0046 \mu \mathrm{m}$ (continuum opacity minimum) using a 16-bit $256 \times 256$ pixel CCD Rockwell Nicmos III (Collados et al. 1997), with an image scale of 0.362 pixel $^{-1}$ and an exposure time of $50 \mathrm{~ms}$. The frames were taken at regular $3 \mathrm{~s}$ intervals.

The influence of chromatic aberration of the telescope's lens inside the two observing bands was previously calculated and discarded as a possible source of errors.

Bursts of 10 images were obtained at spatial intervals of 10 degrees from the disc centre out to the very limb. This processes was repeated on seven different days so that in total 70 images at each heliocentric angle are available. Figure 1 shows a sample of images taken at different heliocentric positions, $\mu$, in the two spectral ranges.

The SVST system accurately specifies the heliographic coordinates of each observed disc position, thus allowing the determination of the heliocentric position $\mu$ for each set of observations. In addition, for positions near the solar limb (i.e. $\mu<0.4) \mu$ has been derived directly by measuring the distance from the limb, which allows a more precise determination in the range where the positional uncertainties are more critical.

Standard flat-field and dark-current correction were applyed to all the images. The noise filtering and the correction for the theoretical point spread function (PSF) of the telescope were performed simultaneously by means of a Wiener filter. To eliminate the gradients due to the CLV of the quiet Sun mean intensity, the images were divided by a first-order polynomial fit. 
Table 1. Effective continuum formation heights $z_{\mathrm{c}}(\mathrm{km})$.

\begin{tabular}{lccc}
\hline \hline$\mu$ & $z_{\mathfrak{c}}(0.80 \mu \mathrm{m})$ & $z_{\mathfrak{c}}(1.55 \mu \mathrm{m})$ & $\Delta z_{\mathrm{c}}$ \\
\hline 1 & 44 & 4 & 40 \\
0.85 & 54 & 10 & 44 \\
0.7 & 66 & 17 & 49 \\
0.6 & 76 & 23 & 53 \\
0.5 & 87 & 30 & 57 \\
0.4 & 102 & 40 & 62 \\
0.3 & 122 & 53 & 69 \\
0.2 & 151 & 74 & 77 \\
0.1 & 201 & 112 & 89 \\
\hline
\end{tabular}

\section{Response functions}

The images analysed are obtained at different heliocentric angles and observing wavelengths. As mentioned above, this implies radiation coming from different layers of the Sun. In order to interpret our observations correctly it is interesting to characterize from which layers of the solar atmosphere the radiation that we observe comes from. This has been performed by means of the response functions (RFs) to the temperature. The RFs are computed by changing the temperature value in a given layer of the photosphere and estimating how the emergent intensity changes. This allows us to characterize the relative contribution of each layer to the total emergent intensity for each wavelength and heliocentric angle. We have computed the $\mathrm{RF}$ by applying the three-dimensional radiative transfer code developed by Fabiani Bendicho \& Trujillo Bueno (1999) to the mean quiet photosphere HSRA (Gingerich et al. 1971). Figure 2 shows the RFs obtained for $\lambda 0.8 \mu \mathrm{m}$ (upper panels) and $\lambda 1.55 \mu \mathrm{m}$ (lower panels) at different heliocentric angles: $\mu=1,0.85,0.7,0.6,0.5,0.4,0.3,0.2$, and 0.1 . In both panels of Fig. 2 the RFs with their main contributions in higher layers represent positions nearer the solar limb.

We define the effective formation heights of the continua, $z_{\mathrm{c}}$, as the positions of the centroids of the response functions. Table 1 shows the $z_{\mathrm{c}}$ values obtained, where $z=0$ corresponds to $\tau_{0.5}=1$. The last column lists the difference in formation heights: $\Delta z_{\mathrm{c}}=z_{\mathrm{c}}(0.80)-z_{\mathrm{c}}(1.55)$. As shown in the table, for larger heliocentric angles (smaller $\mu$ values) the differences in formation heights get larger, which means that the information we obtain comes from more separated layers.

\section{Centre-to-limb variation of quiet granulation contrast}

The contrast, $\Delta I_{\mathrm{rms}}$, of quiet granulation varies from the centre to the limb. The study of this variation is interesting since it gives an idea of the temperature stratification of the solar atmosphere. Many investigations have been performed in this direction and the results obtained by different authors can be grouped into two tendencies. Some authors find that the contrast increases from the disc centre out to $\mu=0.6$ and then decreases (e.g. Rodríguez Hidalgo et al. 1992 for equatorial CLV) while others find a monotonic decrease of the granulation

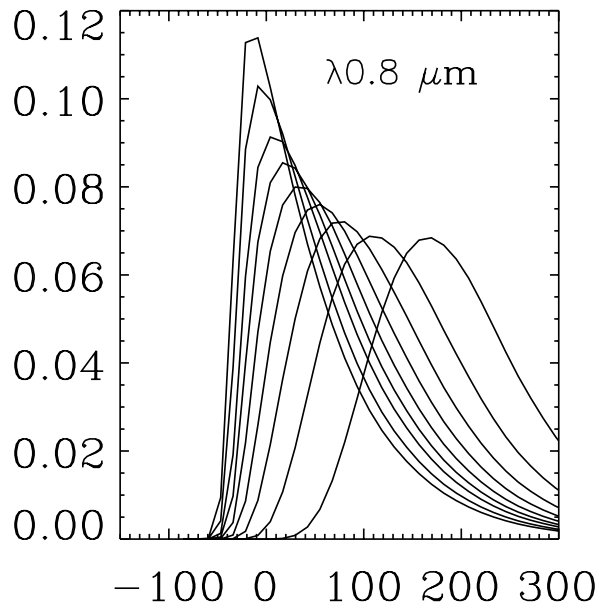

0
01
1
0
0
01
0
0
11

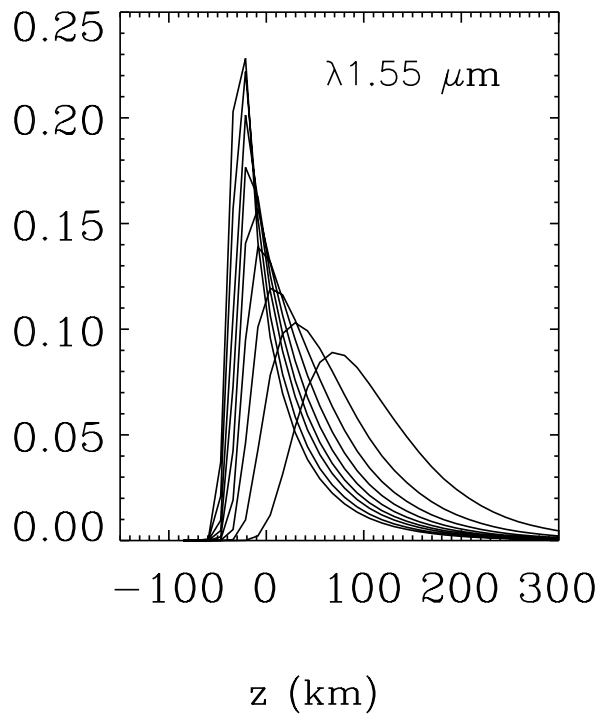

Fig. 2. Response functions to the temperature in the model of a mean quiet photosphere HSRA (Gingerich et al. 1971) at $\lambda 0.8 \mu \mathrm{m}$ (upper panel) and $\lambda 1.55 \mu \mathrm{m}$ (lower panel) at the following disc positions: $\mu=$ $1,0.85,0.7,0.6,0.5,0.4,0.3,0.2$, and 0.1 .

contrast from the centre to the limb (e.g. Wilken et al. 1997; Sánchez Cuberes et al. 2000).

In this paper, the CLV of the $\Delta I_{\mathrm{rms}}$ of quiet granulation has been computed for the $1.55 \mu \mathrm{m}$ and $0.8 \mu \mathrm{m}$ images. The bestquality images at each heliocentric angle have been chosen for this purpose, and the resulting CLV curves normalized to the disc centre value are shown in Fig. 3. The solid line corresponds to $0.8 \mu \mathrm{m}$ images and the dashed line to the $1.55 \mu \mathrm{m}$ images. For the sake of comparison the CLV of the quiet granulation contrast at $0.6708 \mu \mathrm{m}$ from Sánchez Cuberes et al. (2000) and at $1.65 \mu \mathrm{m}$ from Turon \& Léna (1973) are also included in the figure (three dot-dashed). The obtained values of the contrast at the disc centre were $6.1 \%$ for $0.8 \mu \mathrm{m}$ and $2.9 \%$ for $1.55 \mu \mathrm{m}$. The last value is favourably compared to previous determinations by Turon \& Léna (1973): 1.5\% and Koutchmy (1994): $2.07 \%$. 


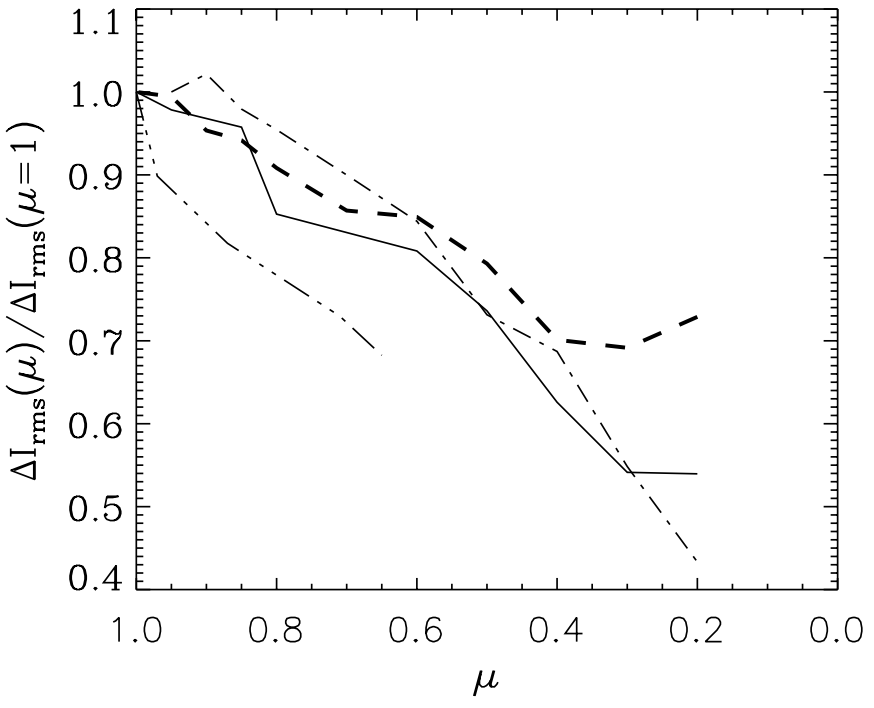

Fig. 3. CLV of the granulation contrast at different wavelengths normalized to the disc centre. $0.8 \mu \mathrm{m}$ solid line, $1.55 \mu \mathrm{m}$ dashed, $1.65 \mu \mathrm{m}$ three dot-dashed (Turon \& Léna 1973), $0.67 \mu \mathrm{m}$ dot-dashed (Sánchez Cuberes et al. 2000).

It should be borne in mind that in the present study the images could not be corrected for the degradation caused by the terrestrial atmosphere which results in the underestimation of the contrast values. Let us make a simple estimate of this degradation under typical seeing conditions characterized by a mean value of the Fried parameter $r_{\mathrm{o}}=11 \mathrm{~cm}(\lambda 0.43 \mu \mathrm{m})$, as measured at the Dutch Open Telescope situated near the SVST (Sobotka \& Sütterlin 2001). Since $r_{\mathrm{o}} \sim \lambda^{6 / 5}, r_{\mathrm{o}} \sim$ $23 \mathrm{~cm}$ at $\lambda 0.8 \mu \mathrm{m}$ and $r_{\mathrm{o}} \sim 51 \mathrm{~cm}$ at $\lambda 1.55 \mu \mathrm{m}$. Denker et al. (1999) reported for Big Bear typical values of $30 \mathrm{~cm}$ at $1.56 \mu \mathrm{m}$. Summarizing, these values characterize high image quality expected in long-exposure frames taken with an aperture of $48 \mathrm{~cm}$. Because our exposures are short $(25$ or $50 \mathrm{~ms})$ and only the best frames are selected, the image degradation due to the atmosphere is minor as compared to that caused by the instrument.

In Fig. 3 it can be observed that, on average, the decrease in the CLV curve of the granulation contrast for $\lambda 1.55 \mu \mathrm{m}$ is not as steep as for $\lambda 0.8 \mu \mathrm{m}$ and $\lambda 0.67 \mu \mathrm{m}$. It would be interesting to know to what extent this behaviour is a physical phenomenon or is caused by the distinct cut-off frequencies of the telescope for the different observing wavelengths. In order to check this we have performed a simple numerical experiment. A high-resolution image of granulation at the disc centre obtained in excellent seeing conditions with the SVST during another observing campaign at $\lambda 0.4507 \mu \mathrm{m}$ was chosen. The image was deconvolved with the instrumental profile of the telescope and denoised with an optimum filter, resulting in a restored reference image with a granulation contrast of $10.4 \%$ and a cut-off frequency of $v_{0.45}=5.2 \mathrm{arcsec}^{-1}$. This image was then rebinned on one of its axes to different sizes, thus simulating the observed foreshortening (obviously, the radiative effects are not included in this simulation). The foreshortened images have been filtered in the Fourier domain by means of low-passband filters decaying smoothly to zero at the cut-off of the telescope at $\lambda 0.8 \mu \mathrm{m}\left(v_{0.8}=2.9 \mathrm{arcsec}^{-1}\right)$ and $\lambda 1.55 \mu \mathrm{m}$

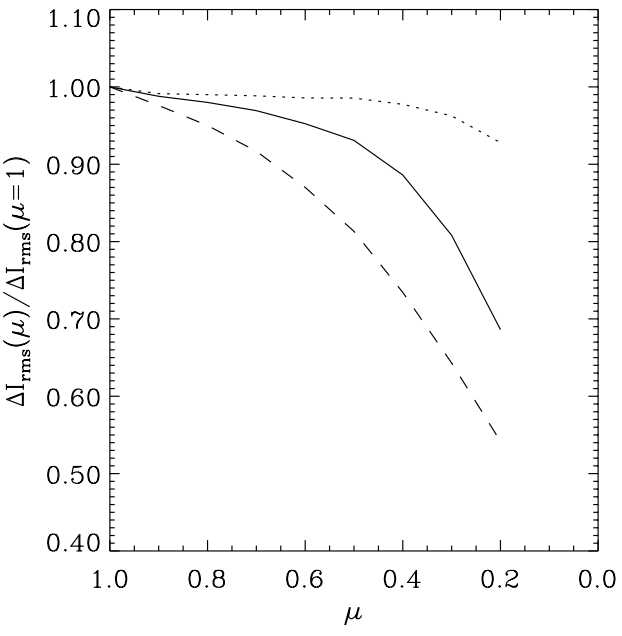

Fig. 4. Computed CLV of granulation contrasts in artificially foreshortened (dotted line) images at $\lambda 0.4507 \mu \mathrm{m}$, filtered with a cut-off at $v_{0.8}$ (solid line) and with a cut-off at $v_{1.55}$ (dashed line). See the text for a detailed explanation.

$\left(v_{1.55}=1.5 \operatorname{arcsec}^{-1}\right)$, respectively. The smooth transition to zero in the filters prevents spurious oscillations in the images caused by the Gibbs phenomenon, and all the frequencies beyond the respective cut-offs were irretrievably lost.

Figure 4 shows the resulting CLV of the contrast in the images with the cut-off at $v_{0.8}$ (solid line) and at $v_{1.55}$ (dashed line), all normalized to the value at the disc centre. The dotted line represents the normalized CLV of the contrast in the foreshortened reference image. This line reveals that the foreshortening by itself hardly affects the contrast of the reference image with a cut-off frequency at $v_{0.45}$ far beyond $v_{0.8}$ and $v_{1.55}$. On the contrary, the images filtered with a cut-off at $v_{1.55}$ and $v_{0.8}$ do suffer a steep loss of contrast from the centre to the limb. Nevertheless, this loss of contrast is more pronounced for images filtered with a cut-off at $v_{1.55}$ than for those filtered with a cut-off at $v_{0.8}$. This is due to the power transfer from small to larger spatial frequencies as we approach the solar limb, which causes the cut-off at $v_{0.8}$ to preserve more power than that at $v_{1.55}$.

In Fig. 3 it can be seen that the observed CLV curve decreases more steeply for $\lambda 0.8 \mu \mathrm{m}$ than for $\lambda 1.55 \mu \mathrm{m}$. Thus, we can conclude that the CLV curve of the granulation contrast diminishes at a higher rate for shorter wavelengths, and that this higher rate of decrease is underestimated due to the effect of the telescope. The origin of these different contrast tendencies can be understood by looking at the response functions of an HSRA atmosphere. The last column of Table 1 shows that the difference in formation height, $\Delta z_{\mathrm{c}}$, increases towards the limb. This is due to a larger increase of $z_{\mathrm{c}}(0.80 \mu \mathrm{m})$ than of $z_{\mathrm{c}}(1.55 \mu \mathrm{m})$ when approaching the limb. Since $\delta \Delta T / \delta \tau$ diminishes with height in the photosphere and contrast is mainly dependent on $\delta \Delta T / \delta \tau$, the contrast will decrease more for $\lambda 0.8 \mu \mathrm{m}$ than for $\lambda 1.55 \mu \mathrm{m}$. The fact that the contrast diminishes monotonically from the centre to the limb for all the observed wavelengths imply that the gradient of temperature decreases steeply with height in the photosphere. If $\delta \Delta T / \delta \tau$ were to diminish less steeply, the observed CLV curve of granulation contrast would 
peak somewhere between the disc centre and the limb (Kneer 1984). However, the obtained CLV curves decrease less steeply in the present work than in the one of Turon \& Léna (1973), performed also in the infrared. This points to a steeper decrease of the $\Delta \mathrm{T}$ associated to the granulation in their work than in the present one.

For $\mu<0.4$ in the $\lambda 1.55 \mu \mathrm{m}$ images and for $\mu<0.3$ in the $\lambda 0.8 \mu \mathrm{m}$ ones, the granulation contrast shows a tendency to increase. Several authors have found an increase in contrast for positions near the limb (Edmonds 1960; Keil 1977; Durrant et al. 1983). This increase could be caused, at least partially, by network bright points whose intensity increases towards the limb and peaks around $\mu=0.2-0.4$ (Auffret \& Muller 1991; Sánchez Cuberes et al. 2002). Other observational effects, specially in $1.6 \mu \mathrm{m}$, like the reduced spatial resolution, difficulties in excluding the bad pixels and signal to noise ratio should also be considered.

\section{Granular and intergranular areas}

The available photometric data allow us to perform a morphological study of the observed images at the working wavelengths. The statistical determination of granular and intergranular sizes are of great importance for the characterization of granulation and comparison with the outputs of the theoretical models.

In order to get statistically representative data, all the images obtained at each heliocentric angle have been included in the present study. To identify the granules, a granule-finding algorithm was used. Images were filtered with a high-passband filter in order to remove large-scale structures other than granulation, such as p-modes, mesogranulation, etc. Following Sánchez Cuberes et al. (2000), the filter was constructed as:

$F(k)=1-\exp ^{-a^{2} k^{2}}$,

where $k$ is the spatial wavenumber and $a$ is a constant proportional to the width of the band to be filtered. In the present study the structures larger than $3 \mathrm{Mm}^{-1}$ have been filtered out.

The technique of segmentation of a granular field based on the use of a filter similar to (1) in combination with a certain threshold was proposed by Roudier \& Muller (1986). Hirzberger et al. (1997) compared this method with the segmentation based on the determination of inflection points at the granular borders and found that the results obtained by both methods were in a very good agreement.

The granule-finding algorithm defines granules as connected regions with intensities greater than that of the mean quiet photosphere (normalized to unity) plus 0.1 standard deviation for each image. This process was performed separately for each filtered image at all the heliocentric angles studied. The fact that the granules are defined as a function of the standard deviation of each image allows us to use a uniform criterion for all the images, independently of their heliocentric angles. The threshold parameter was chosen to produce the best agreement between granule recognition by visual inspection and identification with the granule-finding algorithm. The intergranular lanes were defined following a geometrical criterion. The regions not identified as granules by the granule-finding algorithm are the intergranular lanes. A granule and its corresponding portion of intergranular lane forms a granular cell. Granular cells were defined by setting their perimeters in the middle of intergranular lanes. As discussed in Sánchez Cuberes et al. (2000), radiative effects make the granule determination uncertain when approaching the limb. Thus, only the data ranging from $\mu=1$ to $\mu=0.6$ will be used in order to study the size distribution of granules and intergranules.

Once granules and intergranules have been identified, histograms of their areas can be computed. We obtained that smaller granules are detectable at $\lambda 0.8 \mu \mathrm{m}$ as compared with $\lambda 1.55 \mu \mathrm{m}$. This is logical, since the resolution of the images decreases considerably for the $\lambda 1.55 \mu \mathrm{m}$ images. The histograms for each wavelength also change from the disc centre to $\mu=0.6$, the number of larger granules increasing for larger heliocentric angles, that is, for higher layers in the photosphere. The mean granular size is found to vary from $1.25 \operatorname{arcsec}^{2}$ at the disc centre to $2 \operatorname{arcsec}^{2}$ at $\mu=0.6$ in the $\lambda 0.8 \mu \mathrm{m}$ images and from $1.24 \operatorname{arcsec}^{2}$ to $1.85 \operatorname{arcsec}^{2}$ in the $\lambda 1.55 \mu \mathrm{m}$ ones. Apart from radiative effects, this could be due to the expansion of granules with height in the photosphere. Moreover, a higher efficiency of the large granules in penetrating higher in the photosphere would cause a relative increase in the large granules in the upper layers of the photosphere. In parallel, the less efficient small granules would tend to disappear lower down in the photosphere, which would explain the observed difference between the granular area histograms at different heliocentric angles. Similar histograms of areas for intergranular lanes show the same behaviour.

The granular filling factor (GFF) is defined as the percentage of area covered by granules, i.e. the relative amount of granular area in a given region. Figure 5 presents the CLV of the GFF (solid line) at $0.8 \mu \mathrm{m}$ (upper panel) and $1.55 \mu \mathrm{m}$ (lower panel), together with a second order polynomial fit to the observed trend (dashed line). Vertical bars represent the rms of the dispersion of the measurments at each disc position. The absolute values of the GFF at $\lambda 0.8 \mu \mathrm{m}$ and $\lambda 1.55 \mu \mathrm{m}$ cannot be compared due to the different spatial resolutions. Nevertheless, the CLV of the GFF at each wavelength can give valuable information about the variation of the relative amount of granules and intergranular lanes with height in the photosphere. When observing from the centre to the limb at $\lambda 0.8 \mu \mathrm{m}$, the relative amount of granular area seems to decrease up to a certain $\mu$ and then increases again. This change occurs at $\mu=0.5$. At $\lambda 1.55 \mu \mathrm{m}$, the CLV of the GFF is noisier and no minimum of the GFF can be observed at any disc position. Even though radiative effects impede granule identification at positions near the solar limb, these positions are also included in Fig. 5. The reason is that a relative decrease in the intergranular lanes near the limb is also found by visual inspection of the images.

\section{Penetration of overshooting granules}

An accurate study of the penetration of temperature fluctuations associated with granulation across the photosphere requires spectroscopic measurements. Nevertheless, photometric images of the extreme limb also offer a chance to estimate this 


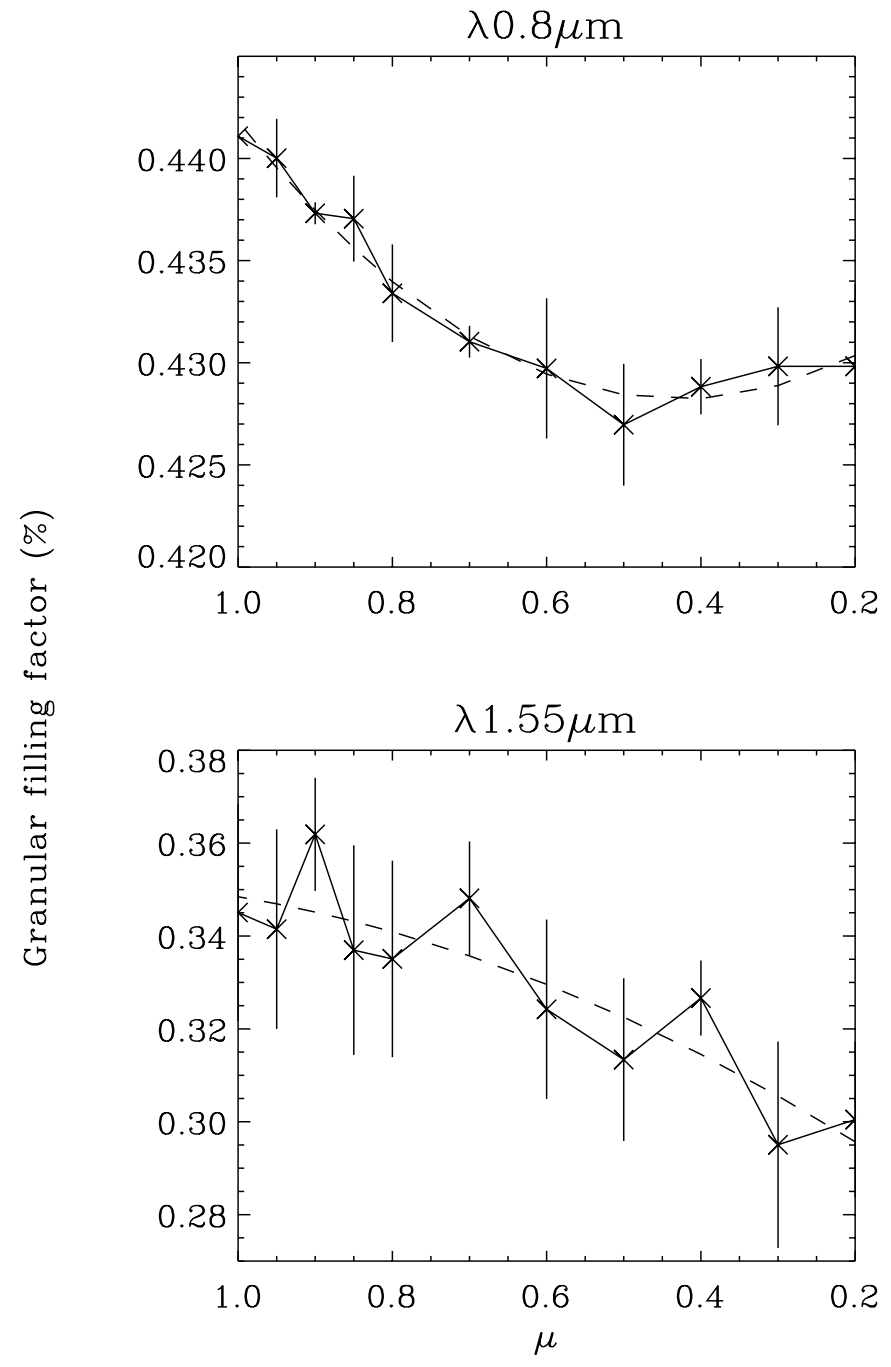

Fig. 5. Variations of granular filling factor with disc position (solid line), second order polynomial fit (dashed line), and rms bars.

height extent by measuring the distance from the limb to which brightness structures can be recognized.

In order to perform this study, the best burst of images containing the solar limb parallel to the $y$-axis of the image was selected. In the $1.55 \mu \mathrm{m}$ images it was impossible to perform a reliable analysis of the data at the very limb due to the low signal-to-noise ratio. Therefore, in this section only the data at $0.8 \mu \mathrm{m}$ will be analysed.

The procedure used to detect structures near the solar limb was performed as described in Sánchez Cuberes et al. (2000). In the following, we will summarize the main steps.

The limb was defined as the inflection point in each row of the image. Due to the distortions induced by terrestrial atmospheric turbulence, the inflection points produced a wavy border to which a straight line was fitted. This line was considered as the origin for measuring distances to the limb. As mentioned above, bursts of ten images were acquired for each observing position. The ten images selected were aligned and averaged in order to improve the signal-to-noise ratio. Then, starting from $d=0$. $^{\prime} 083$ and at intervals of $\Delta d=0 .{ }^{\prime} 083$, scans $34{ }^{\prime \prime} 8$ long parallel to the limb were obtained. The one-dimensional power spectrum of each scan was computed and smoothed by a

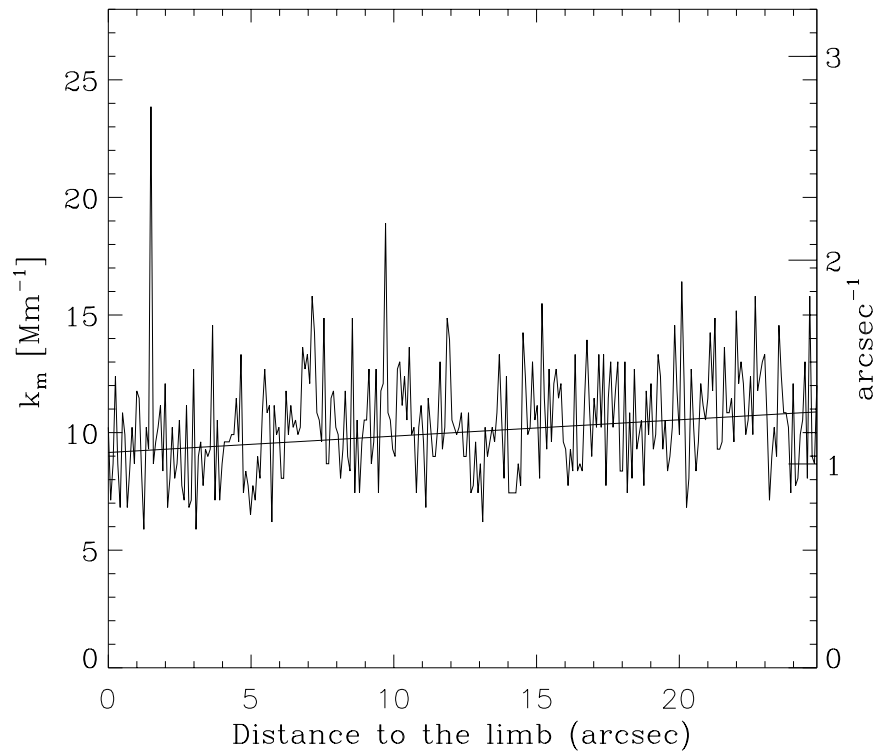

Fig. 6. Variation with distance to the solar limb of the frequency $k_{\mathrm{m}}$ where the one-dimensional power spectra join the noise level in the $\lambda 0.8 \mu \mathrm{m}$ images.

five-point width running box. The noise level of each power spectrum was estimated as the mean power beyond the cut-off frequency of the telescope at $\lambda 0.8 \mu \mathrm{m}$. The frequency $k_{\mathrm{m}}$ at which the signal of the smoothed power spectrum merges with the noise level was then found for each scan. Figure 6 presents the values of $k_{\mathrm{m}}$ obtained as a function of limb distance. The solid straight line is a linear fit to the $k_{\mathrm{m}}$ values obtained, computed by recurrently removing the values lying beyond $2 \sigma$. As seen in the figure, smaller (i.e. higher-frequency) structures are detectable, on average, as moving from the limb towards the disc centre.

Since the distances to the limb are measured with an oversampling, we will consider here that the minimum distance at which structures are detectable is the diffraction limit of the telescope at $\lambda 8000 \AA$, this is, $d=00^{\prime \prime} 32$. We can conclude that frequencies of $1.05 \operatorname{arcsec}^{-1}$ are detectable up to the very limb $\left(d=00^{\prime} .32\right)$. This corresponds to spatial scales of 0.96 , which sets an upper limit to the smallest structures detectable at the very limb. Smaller structures could exist at this position and not necessarily be revealed in the power spectrum as a consequence of the one-dimensional treatment of the data. As discussed in Sánchez Cuberes et al. (2000), the observed structures are probably due neither to the solar oscillations nor to the network bright points.

If we take an Eddington-Barbier approximation for the formation height of the continuum $(\tau=\cos \theta)$ and a conversion from $\tau$ to geometrical height as published by Nelson (1978), the structures observed at $d=0$ '.32 imply temperature fluctuations up to $z \approx 220 \mathrm{~km}$. Nevertheless, the computation of the response functions defines a broader interval. This value can be compared with those obtained by other authors using mainly spectroscopic methods (see Table 2). Note that the penetration height determined here is among the highest in the literature. However, it must be kept in mind that the method used in the present section is not able to assure that the detected structures are really granulation. 
Table 2. Height, $z$, above $\tau=1$, where the temperature fluctuations vanishes or change the sign.

\begin{tabular}{lcc}
\hline \hline Authors & $z(\mathrm{~km})$ & Remark \\
\hline Altrock \& Musman (1976) & $40-130$ & Model dependent \\
Komm et al. (1990) & 170 & \\
Bendlin \& Volkmer (1993) & 265 & \\
Espagnet et al. (1995) & $60-90$ & P-modes filtering \\
Krieg et al. (1999) & 100 & Speckle reconstruction \\
Present work & 220 & \\
\hline
\end{tabular}

\section{Conclusions}

A study of the CLV of the granulation contrast in quiet regions has been performed at $\lambda 0.8 \mu \mathrm{m}$ and $\lambda 1.55 \mu \mathrm{m}$. We have found a monotonic decrease in the contrast at both wavelengths from the centre to $\mu \approx 0.3$. The decrease is steeper at $\lambda 0.8 \mu \mathrm{m}$ than at $\lambda 1.55 \mu \mathrm{m}$. The fact that the granulation contrast does not peak somewhere between the disc centre and the limb implies a strong decrease of the temperature fluctuations with height in the solar atmosphere (Kneer 1984).

The CLV of granular areas has also been investigated. We find an increase in the granular area with heliocentric angle. Mean granular sizes are found to vary from $1.25 \operatorname{arcsec}^{2}$ at the disc centre to $2 \operatorname{arcsec}^{2}$ at $\mu=0.6$ in the $\lambda 0.8 \mu \mathrm{m}$ images and from $1.24 \operatorname{arcsec}^{2}$ to $1.85 \operatorname{arcsec}^{2}$ at $\lambda 1.55 \mu \mathrm{m}$. This increase could be caused by the higher efficiency of the larger granules in penetrating to higher layers in the photosphere. Several authors have found this higher efficiency of the larger granules (e.g., Komm et al. 1990; Nesis et al. 1993), however controversy still exists around this topic. Intergranular lanes are also found to increase their area with heliocentric angle. The granular filling factors have also been computed. We find, in the $\lambda 0.8 \mu \mathrm{m}$ images, a decrease in the GFF with heliocentric angle out to $\mu=0.5$ and then an increase of the GFF out to the limb. In the $\lambda 1.55 \mu \mathrm{m}$ images, the CLV curve of the GFF is noisier and no clear minimum is detected.

Analysis of one-dimensional scans at the extreme limb have revealed that structures as small as 0.96 are detectable out to the very limb. Using a simple Eddington-Barbier approximation, this implies that the temperature fluctuations associated with granulation penetrate at least up to $z \approx 220 \mathrm{~km}$ above $\tau=1$ in the solar photosphere.

In future papers we shall proceed to the numerical simulation of the observations allowing comparison with models of photospheric convective structures.

Acknowledgements. We thank the referee for useful comments and suggestions. We thank also P. Fabiani Bendicho and J. Trujillo Bueno for providing the code to compute the response functions. The support provided by R. Kever and G. Hosinsky during the observations is gratefully acknowledged. This paper has been revised for English and style by the Scientific Editorial Service of the IAC. The SVST is operated on the island of La Palma by the Royal Swedish Academy of Sciences at the Spanish Observatorio del Roque de los Muchachos of the Instituto de Astrofísica de Canarias. This work was partially funded by the Spanish DGES project 95-0028 and PNAYA2001-1649, by the Grant Agency of the Academy of Sciences of the Czech Republic (grant A-3003903), and by the Czech ASCR Key project K-2043105.

\section{References}

Auffret, H., \& Muller, R. 1991, A\&A, 246, 264

Bendlin, C., \& Volkmer, R. 1993, A\&A, 278, 601

Collados, M., Joven, E., Fuentes, F. J., et al. 1997, in Advances in the Physics of Sunspots, ed. B. Schmieder, J. C. del Toro Iniesta, \& M. Vázquez (San Francisco, ASP), ASP Conf. Ser., 118, 361

Denker, C., Spirock, T. J., Goode, P. R., \& Wang, H. 1999, in High Resolution Solar Physics: Theory, Observations, and Techniques, ed. T. R. Rimmele, K. S. Balasubramanian, \& R. R. Radick (San Francisco, ASP), ASP Conf. Ser., 183, 124

Durrant, C. J., Mattig, W., \& Nesis, A. 1983, A\&A, 123, 319

Edmonds, F. N. 1960, ApJ, 131, 57

Espagnet, O., Muller, R., Roudier, Th., Mein, N., \& Mein, P. 1995, A\&AS, 109, 79

Fabiani Bendicho, P., \& Trujillo Bueno, J. 1999, in Proc. 2nd Solar Polarization Workshop: Solar Polarization, ed. K. N. Nagendra, \& J. O. Stenflo (Dordrecht: Kluwer), 219

Gezari, D., Livingston, W., \& Varosi, F. 1999, in High Resolution Solar Physics: Theory, Observations, and Techniques, ed. T. R. Rimmele, K. S. Balasubramanian, \& R. R. Radick, ASP Conf. Ser., 183, 559 (San Francisco, ASP)

Gingerich, O., Noyes, R. W., Kalkofen, W., \& Cuny, Y. 1971, Sol. Phys., 18, 347

Hirzberger, J., Vázquez, M., Bonet, J. A., Hanslmeier, A., \& Sobotka, M. 1997, ApJ, 480, 406

Keil, S. L. 1977, Sol. Phys., 53, 359

Keil, S. L., Kuhn, J., Lin, H., \& Reardon, K. 1994, in Infrared Solar Physics, ed. D. M. Rabin, J. T. Jefferies, \& C. Lindsey (Dordrecht: Kluwer), 251

Kneer, F. 1984, in Small-Scale Dynamical Processes in Quiet Stellar Atmospheres, Sac Peak Obs. Workshop, ed. S. L. Keil, 110, 129

Komm, R., Mattig, W., \& Nesis, A. 1990, A\&A, 239, 340

Koutchmy, S. 1994, in Solar Photosphere: Structure, Convection and Magnetic Fields, ed. J. O. Stenflo (Dordrecht: Kluwer), 81

Koutchmy, S. 1994, in Infrared Solar Physics, ed. D. M. Rabin, J. T. Jefferies, \& C. Lindsey (Dordrecht: Kluwer), 239

Koutchmy, S., \& Lebecq, C. 1986, A\&A, 169, 323

Krieg, J., Wunnenberg, M., Kneer, F., Koschinsky, M., \& Ritter, C. 1999, A\&A, 343, 983

Nelson, G. 1978, Sol. Phys., 60, 5

Nesis, A., Hanslmeier, A., Hammer, R., et al. 1993, A\&A, 279, 599

Rao, Chang-hiu, Jiang, Wen-han, Ling, N., \& Beckers, J. M. 2001, Chinese Astronomy and Astrophysics, 25, 439

Rodríguez Hidalgo, I., Collados, M., \& Vázquez, M. 1992, A\&A, 264, 661

Roudier, T., \& Muller, R. 1986, Sol. Phys., 107, 11

Sánchez Cuberes, M., Bonet, J. A., Vázquez, M., \& Wittmann, A. D. 2000, ApJ, 538, 940

Sánchez Cuberes, M., Vázquez, M., Bonet, J. A., \& Sobotka, M. 2002, ApJ, 570, 886

Scharmer, G., Brown, D. S., Pettersson, L., \& Rehn, J. 1985, Appl. Opt., 24, 2558

Schmidt, W., Deubner, F. L., Mattig, W., \& Mehltretter, J. P. 1979, A\&A, 75, 223

Sobotka, M., Vázquez M., Bonet, J. A., Sánchez Cuberes, M., \& Hanslmeier, H. 2000, ApJ, 544, 1155

Sobotka, M., \& Sütterlin, P. 2001, A\&A, 380, 714

Turon, P. J., \& Léna, P. 1973, Sol. Phys., 30, 3

Wilken, V., de Boer, C., Denker, C., \& Kneer, F. 1997, A\&A, 325, 819 